\title{
Impurities Accumulation on the Surface of Alumina Hydrate Particles in Bayer Technology
}

\author{
GHEORGHE DOBRA ${ }^{1}$, SORIN ILIEV ${ }^{1}$, NICOLAE ANGHELOVICI ${ }^{1}$, LUCIAN COTET $^{1}$, LAURENTIU FILIPESCU2* \\ ${ }^{1}$ Alum SA Tulcea, 82 Isaccei Str. 820228, Tulcea, Romania \\ 2Politehnica University of Bucharest, Faculty of Applied Chemistry and Materials Science, Analytical Chemistry and Environmental \\ Engineering Department, 1-7 Gheorghe P. Polizu Str., 011061, Bucharest, Romania
}

\begin{abstract}
The purity, structural surface, particle dimensions, particle size distribution, and the associated reactivity of chemical and surface properties are the most important and most required properties of alumina hydrate special brands. The purpose of this paper concerns the common metallic impurities accumulation on the surface of alumina hydrate particles, during the sodium aluminates decomposition in liquid phase, during the entire aluminum hydroxide crystallization stage in the Bayer technology.
\end{abstract}

Keywords: Bayer process, alumina hydrate, impurities, scanning microscopy, EDX spectroscopy

Precipitation of alumina hydrate in Bayer technology involves nucleation, agglomeration, and crystal growth, each of these processes taking place in different vessels in the production line. The yield, particle size distribution, particles dimensions, proprieties and purity are deeply dependent on process parameters, and up to certain level, on both organic and inorganic impurities. The concentration of pregnant sodium aluminate solution, caustic ratio in the feeding liquor, seeding ratio, precipitation time, precipitation temperature profile in the production line, the input of hydrate seed and input of aluminate liquor, as well as, the both organic and inorganic impurity concentration, are the most important parameters, which determines the quality of alumina hydrate. Alumina hydrate has a multitude of applications in ceramics, glass, fire retardants, fillers, special semi-calcinated aluminas, pure crystallographic phases, nano and composite materials and other niche domain products. For each application there are required different properties and purity grades [1-4]. If the properties requirement are different form special product to another, the purity standards are permanently raised on the competitive markets.

The paper aim was the study of common metallic impurities accumulation on the surface of alumina hydrate particles, during the sodium aluminates decomposition in liquid phase followed by aluminum hydroxide crystallization stage in the Bayer technology. Also, there were investigated the chemical combinations assumed to be formed in the impurity film on the agglomerated polycrystalline particles, from the firstagglomeration vessel in the production line up to last particle growth and finishing reactor. The study itself does not refer to the chemical identification or chemical analysis of alumina hydrate impurities, but rather to the ways impurities acts in the formation of polycrystalline aggregate, with and without their occlusion in the crystalline product, in any of the stages of crystallization process in Bayer technology. Since the polycrystalline particles are only crystalline phases, the data obtained do not depend on the history of any aggregate, namely, the data refer only to the crystalline surfaces grown in each vessel under typical conditions of over-saturation, concentration and stirring, the level of impurities concentration and additives dosage, etc. Actually, this paper is case study on trial of hydrate production.

\section{Experimental part}

The technique used to analyze the distribution of impurities on the surface of hydrate particles is the scanning microscopy coupled with EDX spectroscopy.

Scanning microscopy is used to study the polycrystalline aggregate morphology, and respectively, the changes in morphology due to the impurities accumulated on the particles surface. Data registration is done digitally, and as a result, the data can be processed in various ways to obtain as much as possible information about the shape and size of the polycrystalline aggregates, sphericity of the polycrystalline aggregates, shape and size of the individual crystals that make up the polycrystalline aggregate, statistical size distribution of individual crystals in the polycrystalline aggregate, the crystal-crystal interfaces morphology, etc.

EDX spectroscopy allows elemental analysis over the scanned surfaces of alumina hydrate particles. Mainly, an electron beam is focused onto the sample surface being studied, and the resulting $X$ radiation is recorded and statistically analyzed to give coherence to the relationship between simultaneous scanned geometric shapes and their surface elemental composition. The EDX analyzer displays information about three important parameters:

- Distribution of the chemical elements on the surface of crystals or polycrystalline aggregate. In the present study we will be considering the following elements, as elements of high interest: $\mathrm{Al}, \mathrm{Na}, \mathrm{Ca}, \mathrm{Mg}, \mathrm{Si}, \mathrm{S}, \mathrm{Fe}$ and C;

- Relative concentrations of the elements taken into account. That is, the sum of all identifiable elements concentrations in a sample is considered 100 percent, and each element contributes with some weight to these 100 percents. In this case, in the alumina hydrate samples, aluminum has the highest weight against the weight of other elements. As a result, we will report the concentrations of all elemental impurities to the aluminum concentration (approximately constant, given the low masses of other elements);

- Possible combinations of a group of elements. These combinations are suggested by the superposition of the $X$ ray dispersion peaks of the tied up elements in the chemical combinations.

All accessible data can be collected at a point, on a linear profile, on a limited part of the scanned surface or on the entire surface illuminated by the electron beam sent 
to the surface of the sample to obtain the photographic image. Concentrations of the elements, more precisely the ratios between them refer to scattered points or scattered selected areas from the total electron beam illuminated surface (different as positions, but correlated with the particles surface concentrations). The electron flow is evenly distributed on the scanned surface, but it does penetrate the sample in depth, not more than $10-100 \mathrm{~nm}$ or exceptionally lum. It should, also, be noted, that the electron flow is focused on the surface of the sample and everything that comes out beyond focusing depth can not be seen. As a result, the ratios between the concentrations of the elements shown are not real ratios in the entire sample mass. But, they only approximate surface ratios. The actual mass concentrations of the sample may be lower or higher and can only be determined by chemical analysis. All data are digitally recorded and can be used independently for any interpretation of the photomicrograph with means compatible with the results of the X-ray spectroscopy.

\section{Results and discussions}

A. Case study over the quality in industrial production of alumina hydrate

The main purpose of these experimental investigations was the efficiency demonstration of scanning microscopy coupled with EDX spectroscopy in the study of alumina hydrate contamination with impurities from the sodium aluminate solution, in crystallization stage from Bayer technology.

\section{Scanning Microscopy}

Scanning microscopy data may be presented in various ways for highlighting certain peculiarities of crystals and crystalline agglomerates. In one variant, the photomicrographs reveal the surface of the crystals in polycrystalline aggregate. In some other variants, the morphology of the edges and the separation bridges between the crystals appear distinctly. Most of variants show the shape, impurities display and their zonal accumulation or structural properties of the particles in all the vessels of the alumina hydrate production line (fig. 1). Moreover, digital data allows segmentation of the image and identification of all individual particles within the structure of polycrystalline aggregate (fig. 2). This is quite a flat-plan 3D projection

Actual particles are differentiated from the voids betw een the particles, and the individual particles can be counted and classified by the projected area size or geometric shape (fig. 2). Further, the segmentation data allows a statistical analysis of the particle size distribution in any individual polycrystalline aggregate (fig. 3). Figure 3

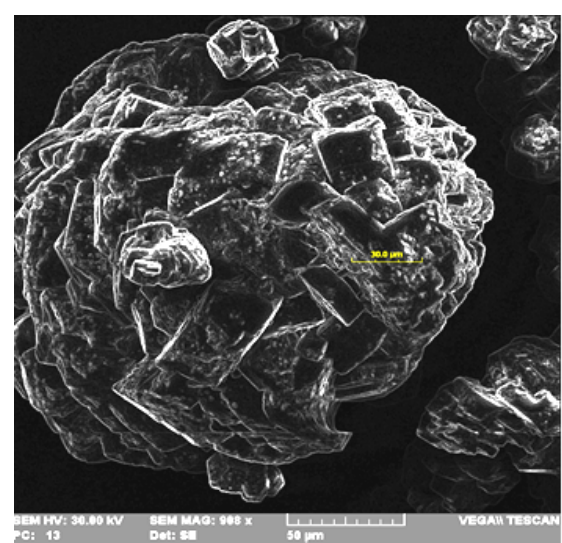

Fig. 1. Sample 16A-Sobel microphotography of the alumina hydrate collected from agglomeration stage

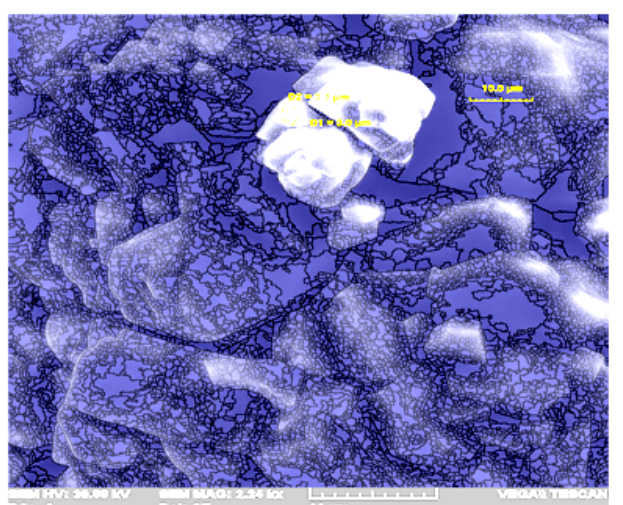

Fig. 2. Sample 5A-Non - milled alumina hydrate production. Segmentation microphotography

shows that the distribution is bimodal with two maximums at 2.05 and $2.48 \mu \mathrm{m}$ and a maximum size of $5.65 \mu \mathrm{m}$, before being fully embedded in larger aggregates. In other words, we may think the data of figure 3 in the sense that any crystal larger than $5.65 \mu \mathrm{m}$ is found in the larger particle structure. All crystals smaller than $5.65 \mu \mathrm{m}$ are randomly placed at the surface of larger particles, more or less attached by bridges to this surface or by bridges to some smaller associations inside the bigger particles.

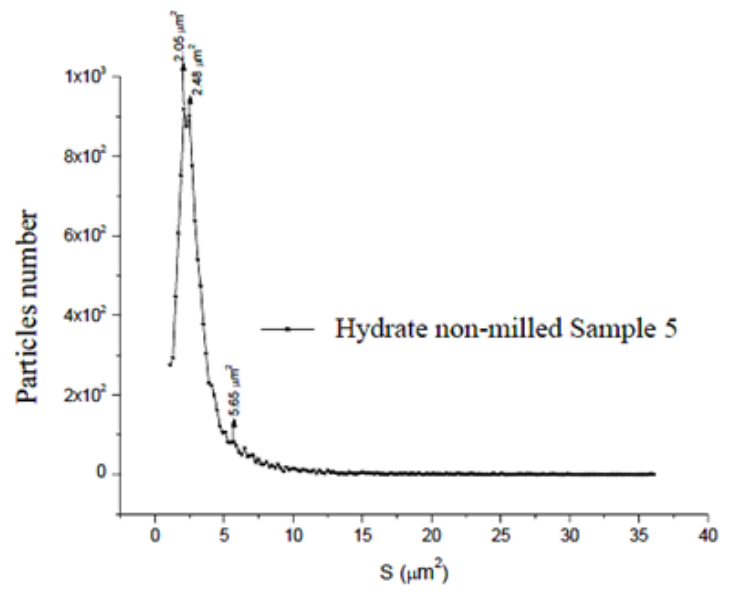

Apparent surface $\mathrm{S}(\mu \mathrm{m})$

Fig. 3. Statistical particle size distribution according to segmentation microphotography

Mainly, the small size of the smallest crystals in polycrystalline particles means a larger area (much larger if we take into account that the maximum share in the finished product belong to particles with tens of microns dimension) exposed to the adsorption of impurities during the growth of usual polycrystalline aggregates. Because this analysis method only refers to the visible surfaces for scanning, it is easily to see that the maximum dimensions on the statistical distribution curve in figure 3 are the dimensions of crystals formed in stages where the samples were collected. Thus, due to this indirect measurement, we can basically find out the dimensions of individual crystals grown in each vessel in the production line, and also the elementary size of crystals separated in hydrocyclones as seeding crystals.

\section{EDX Spectroscopy}

This scanning technique coupled with scanned microscopy offers a much more complex set of information. The data digitization allows the knowledge of impurities distribution on crystalline surfaces, their association in chemical compounds and the origin of these chemical compounds (the place of formation, the place of 
penetration and recycling on the technological line of alumina hydrate crystallization).

\section{Mapping the scanned area}

The first and most remarkable information comes from the mapping of scanned microscopic surface. A second scan of the sample surface, which is superimposed on the photographic image, detects and displays the atoms of all detectable elements by X-ray spectroscopy. Practically, in every pixel of the photographic image there is introduced full information about the elemental composition, given by the X-rays scanning. Data storage is selective for the atoms of all elements chosen as target of any particular interest, and for each of these elements the storage is selective according to their concentrations. As a result, the access to these data is selective. That is, we can select the available data from a point, on a surface or on a linear profile in the photographic image. In the figure 4 there are given the mappings of the samples 5B milled alumina hydrate production. In this type of figures, each element is viewed in a distinctive colour. But in the figure 4, only sulphur was marked.

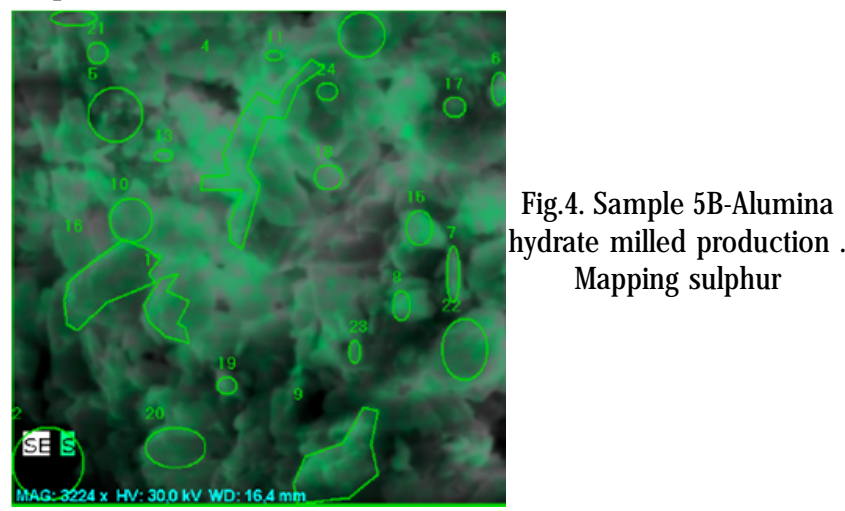

In the figure 5 there are displayed the areas covered with aluminium and magnesium. Figure 5 shows that there are areas covered by each individual element and mixed areas (mixed colours) corresponding to $\mathrm{Mg}-\mathrm{Al}$ combinations. This last information is only qualitative, and must therefore be confirmed by a linear concentration profile passing through one of the mixed colour zones.

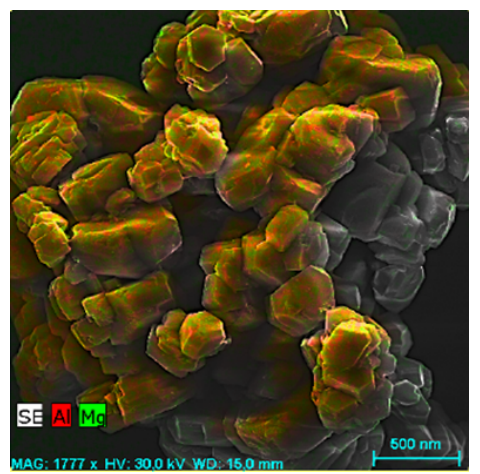

Fig.5. Vizualization of the aluminium and magnesium covered zones from sample $5 \mathrm{~A}$

Digital mapping allows the calculation of relative concentration for each element over any specific chosen areas at the particles surface (fig. 5). Relative concentrations will be expressed as ratios between each element and aluminium as the major element. It is implicitly admitted that each atom of an element tied up or non-tied up in a combination with aluminium with appears in the photograph as totally or partially superimposed over an aluminium atom. At high concentrations (relative), the impurity can completely hide the aluminium atoms. Table 1 lists the whole surface scanning data for samples 5A and 5B. EDX analysis of non- milled and milled and samples enables the identification of occlusions (inside the poly-crystalline alumina hydrate particles) in the collected samples. If there is an occlusion, after milling, the impurities are redistributed to the surface of the new particles formed by milling. Milling means the breaking of larger polycrystalline aggregates into smaller particles and implicitly the increasing of surface available for redistribution of impurities. The evidence that only large particles are divided by milling is the comparison of the samples.

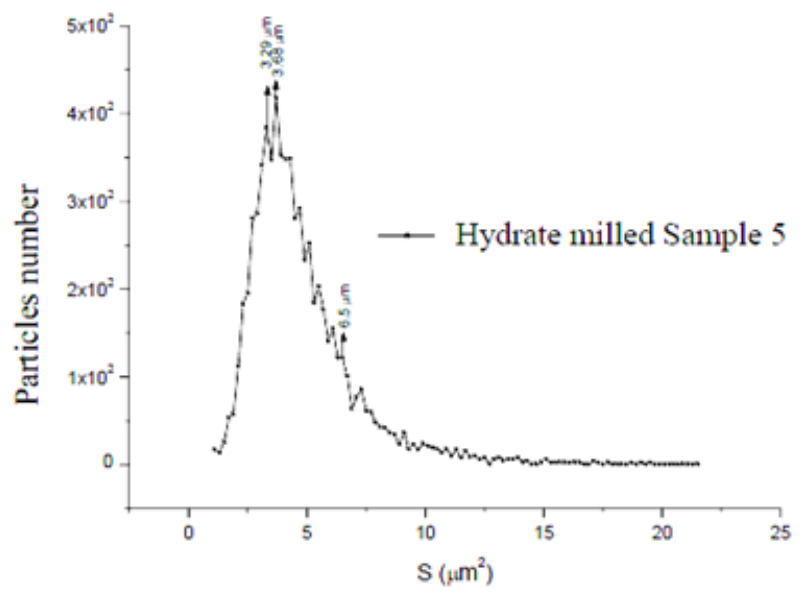

Apparent surface $\mathrm{S}(\mu \mathrm{m})$

Fig. 6. Statistic particle size distribution in sample 5B-milled alumina hydrate production

Statistic particle size distribution computed for the sample 5B (milled alumina hydrate, fig. 6), and the statistic particle size distribution computed for the sample $5 \mathrm{~A}$ (nonmilled alumina hydrate, fig. 3) illustrate the above assumption. It can be seen from the figure 6 that the maximums of the bimodal distribution move to higher values, 3.29 and $3.69 \mu \mathrm{m}$, respectively. In the absence of inclusions, the impurities adsorbed on the surface of nonmilled particles will be redistributed after milling on a larger area. Therefore, the concentration of these impurities will be diminished proportionally with the increases in the available area. If there are some inclusions in the large particles, the concentration of impurities, redistributed after milling, should significantly to increase. As far as figure 7 and table 1 show, none of the elements $\mathrm{Na}, \mathrm{Si}, \mathrm{Ca}$ and $\mathrm{Mg}$ are occluded, as captive drops of mother liquor inside the polycrystalline particle structure.

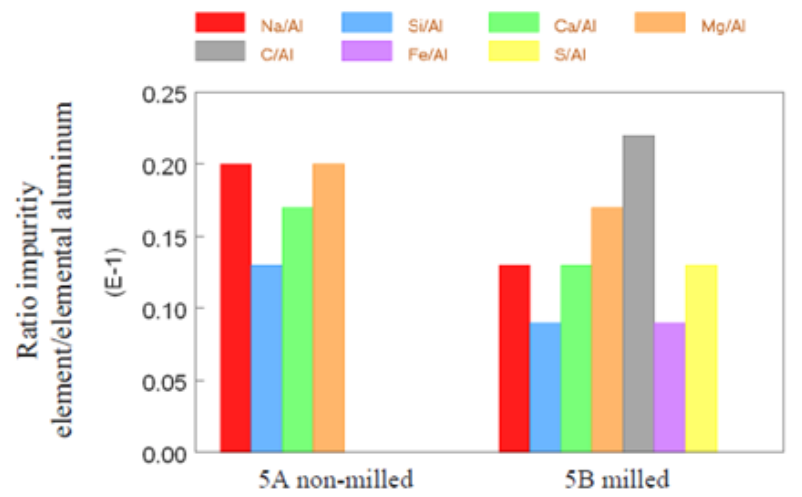

Fig. 7. The surface distribution of impurities after specific area extention by milling

Although there are not much data on carbon, iron and sulphur absorption/adsorption on alumina hydrate particle surface in Bayer mother liquors, some assumptions can be made aboutthe occlusion of these elements from other data processing of the optical and EDX images. According to the present experiments, the sulphur is found in the 
Table 1

RATIOS ELEMENT/ALUMINIUM IN THE ACCUMULATED IMPURITIES ON PARTICLES SURFACES IN SAMPLE 5-ALUMINA HYDRATE PRODUCTION

\begin{tabular}{|l|l|l|l|l|l|l|l|l|}
\hline Sample & $\mathrm{Al}$ & $\mathrm{Na} / \mathrm{Al}$ & $\mathrm{Si} / \mathrm{Al}$ & $\mathrm{Ca} / \mathrm{Al}$ & $\mathrm{Mg} / \mathrm{Al}$ & $\mathrm{C} / \mathrm{Al}$ & $\mathrm{Fe} / \mathrm{Al}$ & $\mathrm{S} / \mathrm{Al}$ \\
\hline 5A Alumina trihidrate non-milled & 5.37 & 0.020 & 0.013 & 0.017 & 0.020 & & & \\
\hline 5B Alumina trihidrate milled & 5.35 & 0.013 & 0.009 & 0.013 & 0.017 & 0.022 & 0.009 & 0.013 \\
\hline
\end{tabular}

SAMPLES OF ALUMINA HYDRATE PRODUCTION QUALITY PREPARED BY DISSOLUTION OF SOME PARTICLES SURFACE MOLECULAR LAYERS

\begin{tabular}{|l|c|c|c|c|c|}
\hline Sample & $\mathrm{Al}$ & $\mathrm{Na} / \mathrm{Al}$ & $\mathrm{Si} / \mathrm{Cl}$ & $\mathrm{Ca} / \mathrm{Mg} / \mathrm{Al}$ & $\mathrm{M}$ \\
\hline 5A Alumina Hydrate non -milled & 5,37 & 0.020 & 0.013 & 0.017 & 0.020 \\
\hline 5B Alumina Hydrate milled & 5,35 & 0.013 & 0.009 & 0.013 & 0.017 \\
\hline 5C Alumina Hydrate nitric acid treated non-milled & 5,45 & 0.011 & 0.009 & 0.013 & 0.015 \\
\hline 5D Alumina Hydrate nitric acid treated milled & 5,38 & 0.015 & 0.013 & 0.017 & 0.020 \\
\hline 5E Alumina Hydrate milled and nitric acid treated & 1.96 & 0.036 & 0.056 & 0.051 & 0.051 \\
\hline
\end{tabular}

sodium aluminate solutions as sulphate ions associated with sodium, calcium and magnesium in alumino-silicates compounds. If sodium, calcium and magnesium are not contained in mother liquors, then neither the sulphate ion is occluded. The carbon as an organic ion is adsorbed on the alumina hydrate particles surface, and itis incorporated into their crystalline network. So, its occlusion plays a minor role in explaining the large carbon content accumulated onto the surface of the milled particles. If some of the carbon content originates from carbonate compounds in mother liquors, then the occlusion of carbonate ions might be explained by the occlusion of sodium, calcium and magnesium carbonates. The same considerations are applying to the sulphur as sulphate ion. Also, the iron is adsorbed onto the surface of alumina hydrate particles surface in a particularly form of hydroxide, irrespective of the crystallization stage of the alumina hydrate. Therefore, its occlusion as drops of mother liquor inside agglomerated particles does not contribute to the impurities accumulation on crystalline surfaces during alumina hydrate crystallization.

\section{B. Recording the impurity data and their use for describing alumina hydrate purity}

The use of scanned sample mapping to analyze how impurities are incorporated into the mass of alumina hydrate.

It has been shown above that the extension of the sample surface by milling does not increase the concentration of the impurities. It was considered that the analyzed sample did not contain occlusions like the usual mother liquor drops inside the polycrystalline structure of particles. Taking advantage of an available and accurate method to measure the relative surface concentrations, the alumina hydrate sample was treated with concentrated nitric acid solution in order to eliminate a significant number of deposited layers on the crystalline surface of the agglomerated particles. After that, so prepared sample was scanned according to the above described methodology. Sample 5A from the table 2 is the raw alumina hydrate collected from the production line and sample 5B is the same material superficially ground for breaking the largest aggregates from the figure 7 . Sample $5 \mathrm{C}$ originated from sample $5 \mathrm{~A}$ treated with nitric acid (20\%) and washed with water. Through this treatment, the $\mathrm{Na}, \mathrm{Si}, \mathrm{Ca}$ and $\mathrm{Mg}$ elements, probably bound as silicates and small amounts of aluminium embedded in the silicate structure, have been removed from the crystalline particles surface. By comparing the data of these two samples, $5 A$ and $5 B$, it was found that parts of each impurity element ware dissolved in the nitric acid and then, were removed from the surface of the crystals. In other words, the alkaline combinations of these elements have been neutralized and dissolved by nitric acid. The remaining material after nitric acid treatment was milled and analyzed again under the new name, sample 5D. As it was shown in the figure 8, the concentrations of all four elements from sample $5 \mathrm{D}$ are greater than those in samples $5 \mathrm{~B}$ and $5 \mathrm{C}$, but lower than those from sample $5 \mathrm{~A}$. As a result, the $5 \mathrm{C}$ sample milling has brought to the surface of crystals in sample 5D new amounts of pre-existing impurities in the bridges between crystals, which were not accessible for the nitric acid, when the sample $5 \mathrm{~A}$ was treated with nitric acid.

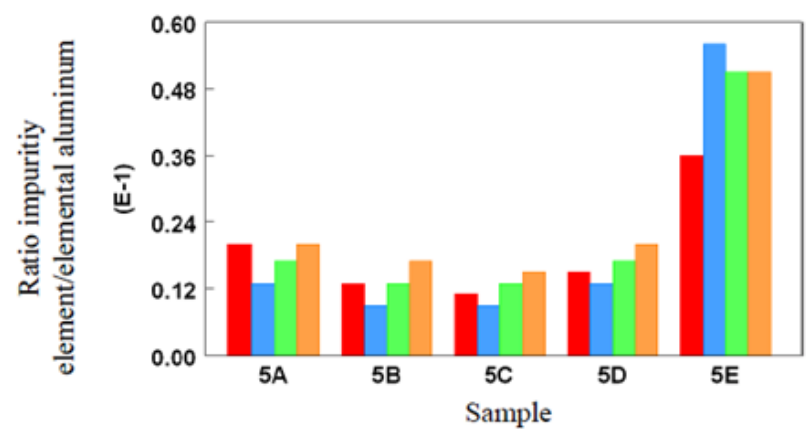

Fig. 8. Surface impurities distribution of the sample 5-alumina hydrate (production) after its processing, in order to identify the occlusions of impurities during the last stage of crystallization

Apparently, these new impurities are also soluble in nitric acid. This will be further demonstrated. Sample 5D was treated again with nitric acid, resulting in sample $5 \mathrm{E}$. The surface impurity concentrations of crystals in sample $5 \mathrm{E}$ are two times larger then concentrations in previous samples. This experiment has shown that in alumina hydrate there are two categories of impurities: nitric acid soluble surface impurities (may be, predominantly sodium, magnesium and calcium silicates/sulphates) and insoluble impurities containing the same elements $\mathrm{Na}, \mathrm{Si}, \mathrm{Ca}$ and $\mathrm{Mg}$, which are bound as alumino-silicates compounds. The capture of these impurities inside alumina hydrate particles, occurs at different stages of crystallization process and at different dimensions of the aggregate particles. Probably, the aluminium - silicates come from desilication stage or red filtration process, as very fine particles. During the agglomeration at a beginning stage of alumina crystallization, when the liquor supersaturating rate is high, and the rate of super-saturation uploading is also high, these fine particles serve as hetero-nuclei at the crystalline surfaces and are quickly attached to the finest crystalline aggregate nuclei (2-2.5 $\mu \mathrm{m}$ in fig. 3). The impurities bound in the form of nitric acid-soluble silicates account for the dowry of sodium aluminate solution. They are adsorbed to the surface of alumina hydrate crystals in any of the postagglomeration stages due to small variations in super- 


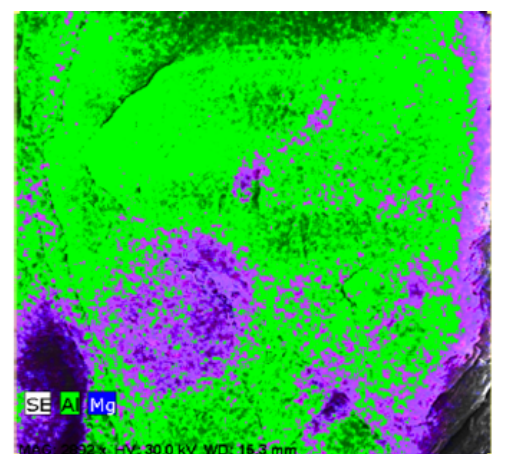

Fig. 9. Layout of MgO clusters on the surface of a calcined alumina crystals

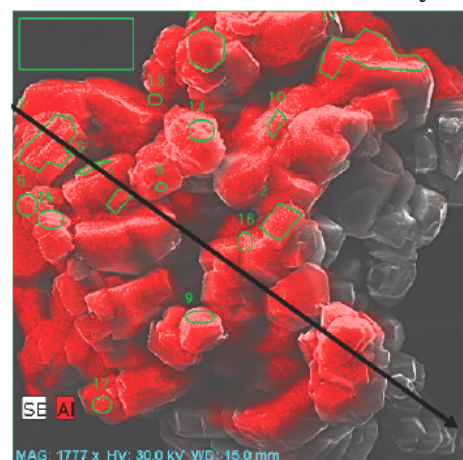

Fig. 10. Hypothetical profile of the impurities concentration

saturation, concentration or temperature. Probably, most of these impurities remain on the surface of the alumina hydrate particles as a result of insufficient or inefficient washing. In conclusion, there are two sources of impurification: a) suspended particles which are not easy removable in the red filtering stage, and b) soluble silicates in the aluminate solution, eventually removed by controlling the crystallization process and by intensive washing. It should also mind about, that each metallic impurity does not individually reach the surface of alumina hydrate particles and that the participation of various compounds incorporating metallic impurities in the contamination process does not exclude selective impurification.

Selective contamination zones detectable by mapping

The impurities are not uniformly distributed over the scanned surface of samples. There are specific areas of impurities, which prove their origin.

Actually, these zones growth has started either from the aluminate solution (the nitric acid soluble salts nucleation at small concentrations) or from the pre-existing impurities as fine crystalline or colloidal particles in the sodium aluminate liquors. Experimentally, there were visualized zone of impurities accumulation and clustering, like magnesium compounds on alumina hydrate surface in the figure 9 .

Identifying chemical combinations including the impurities in alumina hydrate

In very simple cases, the arrays of molecules on the scanned surface of the sample is ordered by a strong action parameter, which implies the exclusion of impurities from the well-formed crystals mass and from inter-particles bridges. In such a case, the overlapping several EDX photographs can be visualized in a selective arrangement of the characteristic elements along one or more concentration profiles. Then, the nature of the existing chemical combinations can be find out from the numbers
Table 3

PURITY CONCENTRATIONS IN ZONE 3 FROM FIGURE 4

\begin{tabular}{|c|c|c|c|c|c|c|}
\hline $\mathrm{a} / \mathrm{Al}$ & $\mathrm{Si} / \mathrm{Al}$ & $\mathrm{Ca} / \mathrm{Al}$ & $\mathrm{Mg} / \mathrm{Al}$ & $\mathrm{C} / \mathrm{Al}$ & $\mathrm{Fe} / \mathrm{Al}$ & $\mathrm{S} / \mathrm{Al}$ \\
\hline 020 & 0.013 & 0.017 & 0.020 & & & \\
\hline 013 & 0.009 & 0.013 & 0.017 & 0.022 & 0.009 & 0.013 \\
\hline 014 & 0.014 & 0.025 & 0.021 & 0.213 & 0.021 & 0.014
\end{tabular}

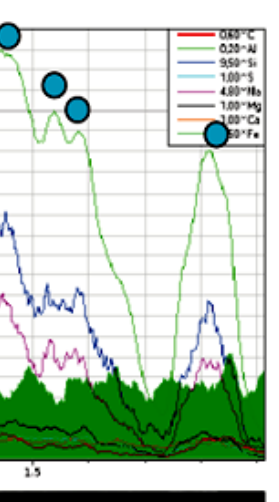

elements aluminum, silicium, sodium and iron in alumina hydrate

of peaks superposing on X-ray spectra of two or more elements.

The reading principle of concentration profile for one or more elements is illustrated in figure 10. Such an example of concentration profiles for elements of interest in the impurities accumulation on the alumina hydrate particles surface is given in the figure 11 . The analysis of the experimental data showed that the existence of free aluminum is normal (it is abot the gibbsite crystals). Also, the data assumes that much of the elements of interest (sodium, magnesium, calcium and silicon) are bound in alumino - silicates, more or less associated. Of course, the nature of the alumino-silicates and their concentration might be easily determined by other more refined methods. But, the scanning microscopy coupled with EDX spectroscopy can bring some supplementary information, because it can discriminate the impurity accumulation on the alumina hydrate at particles surface from impurities accumulation by occlusion. Also, this method is important in Bayer technology, because it can say more precisely, where on the production line the alumina hydrate was impurified.

\section{Conclusions}

The study shows how the impurities participate in the agglomeration and growths processes of polycrystalline aggregates, with and without their occlusion in the final product, the alumina hydrate. The samples were raised from different stages of process in the production line of alumina hydrate by Bayer technology. There were shortly described the methods of samples processing, EDX microphotographic analysis, as well as, the procedures to comprehend and interpret the digital data. Mainly, the digital data were used to find out the mechanisms of impurities accumulation in alumina hydrate final product and the ways to control and prevent the product impurifying process.

\section{References}

1. TEMUUJ IN, J., MACKENZIE, K.J.D., SCHMUCKER, M., SCHNEIDER, H., MCMANUS, J., WIMPERIS, S., J. Eur. Ceramic Society 20, 2000, p. 413 Phase evolution in mechanically treated mixtures of kaolinite and alumina hydrates (gibbsite and boehmite) 
2. ZHU, H.Y., RICHES, D., BARRY J.C., Chem. Mater. 14, nr.5, 2002, p. 2086; $\gamma$-Alumina nano-fibbers prepared from aluminium hydrate with poly(ethylene oxide) Surfactant

3. VIEIRA COELHO, A.C., SANTOS, H.S., KIYOHARA, P.K., PINTO MARCOS, K.N.P., SOUZA SANTOS, P., Mat. Res. 10, nr.2, 2007, p.189; Surface area, crystal morphology and characterization of transition alumina powders from a new gibbsite precursor
4. PETERSEN, M.R., CHEN, A., ROLL, M., JUNG, S.J ., YOSSEF, M., Composites Part B: Engineering, 78, 2015, p.109 Mechanical properties of fire-retardant glass fibber reinforced polymer materials with alumina hydrate filler

Manuscript received: 5.12 .2018 